\title{
Cochinillas presentes en viñedos de Colonia Caroya, Córdoba, Argentina. Incidencia en el cultivo y efectos sobre la calidad de los mostos
}

\author{
Viglianco, A.I.; C.I. Cragnolini, M. Bocco y S.C. Reynoso
}

\begin{abstract}
RESUMEN
En viñedos de Colonia Caroya, Córdoba, se ha observado un incremento de la población de cochinillas a partir de 2001-2002. El objetivo del trabajo fue determinar las especies presentes, su incidencia en viñedos de la zona y sus efectos sobre calidad de mostos. Para identificar las especies se recolectó material vegetal de variedades tintas y blancas; se cuantificó la incidencia y la intensidad de la especie más abundante en 15 viñedos en 2010 y 2011 y se determinó el efecto de las cochinillas sobre la calidad de mostos en las variedades Chardonnay, Merlot y Pinot noir. Se identificaron dos especies de Pseudoccocidae, Planococcus ficus (Signoret) y Pseudococcus viburni (Signoret) que afectan racimos, hojas, brotes, sarmientos y troncos y una de Coccidae, Parthenolecanium persicae (Fabricius) que afecta fundamentalmente hojas, sarmientos y troncos. La especie más abundante es $P$. ficus, con una incidencia en viñedos de $73 \%$ en 2010 y $87 \%$ en 2011. En este período la incidencia en plantas varió de 17 a $28 \%$, y a medida que crece el porcentaje de plantas afectadas lo hace también el de racimos. En racimos con más de $50 \%$ de raquis afectado se observa deterioro de algunos índices de calidad de mostos.
\end{abstract}

Palabras clave: Planococcus ficus, Pseudococcus viburni, Parthenolecanium persicae, viñedos, calidad de mostos.

Viglianco, A.I.; C.I. Cragnolini, M. Bocco and S.C. Reynoso, 2016. Mealybugs present in vineyards of Colonia Caroya, Córdoba, Argentina. Incidence on the crop and effect on must quality. Agriscientia 33 (1): 27-38

\section{SUMMARY}

An increase in mealybug populations has been observed in vineyards of Colonia Caroya, Córdoba, since 2001-2002. The aim of this work was to determine the species present, their incidence on vineyards in the area and their effects on must quality. Species were identified by collecting plant material of red and white grape varieties; incidence and intensity of the most abundant species was quantified in 15 vineyards in 2010 and 2011, and the effect of mealybugs on the must quality of the varieties Chardonnay, Merlot and Pinot noir was 
determined. Two Pseudococcidae species were identified: Planococcus ficus (Signoret) and Pseudococcus viburni (Signoret), which affect clusters, leaves, buds, vine shoots, and stems, and one Coccidae species, Parthenolecanium persicae (Fabricius) mainly affecting leaves, vine shoots and stems. The most abundant species was $P$. ficus, with an incidence in vineyards of $73 \%$ in 2010 and $87 \%$ in 2011. During that period, incidence in plants varied from 17 to $28 \%$ and the percentage of infested grape clusters increased with increasing percentage of infested plants. In clusters with more than $50 \%$ of rachis affected, the deterioration of some quality indices of must is observed.

Keywords: Planococcus ficus, Pseudococcus viburni, Parthenolecanium persicae, vineyards, must quality.

A.I. Viglianco, C.I. Cragnolini y M. Bocco: Facultad de Ciencias Agropecuarias, Universidad Nacional de Córdoba. Av. Valparaíso s/n, Ciudad Universitaria. 5000, Córdoba, Argentina. S.C Reynoso: Bodega La Caroyense S.A., Colonia Caroya, Córdoba, Argentina. Correspondencia a: aviglian@agro.unc.edu.ar

\section{INTRODUCCIÓN}

La industria vitivinícola argentina ha evolucionado considerablemente en los últimos años, ubicándose la Argentina entre los primeros productores de vinos en el mundo. Teniendo en cuenta la superficie implantada, Córdoba es la octava provincia con 278 ha que contribuyen con aproximadamente el $0,12 \%$ de la superficie implantada en el país (INV, 2015). Dentro de la provincia, la principal área productora es Colonia Caroya (Departamento Colón), donde el $97 \%$ de la producción se destina a la elaboración de vinos y jugo de uva. A partir de la temporada 2000-2001 se observó en Colonia Caroya la presencia de cochinillas en plantas aisladas de vid. Las poblaciones se incrementaron en forma notoria desde 2004 en adelante. Las cochinillas constituyen un problema creciente ya que se manifiestan en viñedos de todo el mundo (Pearson \& Goheen, 1996; Bentley et al., 2000; De Borbón et al., 2004; Daane et al., 2008; Mohamed et al., 2012; Miano et al., 2014). En este cultivo se encuentran representantes de las familias Pseudococcidae, Coccidae y Diaspididae (Cordo et al., 2004), y a pesar del incremento de la plaga y los daños que causa en Colonia Caroya no están registradas las especies presentes, su incidencia y su efecto sobre la calidad de los mostos.

Las cochinillas harinosas (Hemiptera: Pseudococcidae) se caracterizan por tener cuerpo blando, claramente segmentado, cubierto con cera pegajosa blanca que se extiende hacia los apéndices (Walton \& Pringle, 2004; Bentley et al., 2008). Las distintas especies causan daño económico. En Estados Unidos se encuentran Planococcus ficus (Signoret), Pseudococcus maritimus (Ehrhorn), Pseudococcus viburni (Signoret) y Pseudococcus longispinus (Targioni-Tozzeti) (Geiger et al., 2001; Daane \& Bentley, 2003; Daane et al., 2004); en España, Pseudococcus citri Risso (Toledo Paños, 2004); en Sud África (Walton \& Pringle, 2004) y México (Fu Castillo et al., 2004), P. ficus. En Chile las más frecuentes son P. viburni (Klopow \& Zaviezo, 2004; Valenzuela, 2008), P. ficus, P. Iongispinus y P. maritimus (González \& Volosky, 2006); en Uruguay, P. ficus, P. citri y P. minor (Granara de Willink et al., 1997) y en Brasil, P. viburni (Morandi Filho, 2008). En la Argentina la especie más difundida es $P$. ficus y se la encuentra en Mendoza (Becerra et al., 2006a), San Juan (Bustos, 2004), Catamarca y Tucumán (Granara de Willink, 2004). También se reporta la presencia de Ferrisia virgata (Cockerell) en La Rioja (Trjapitzin \& Trjapitzin, 1997), Córdoba, Catamarca y Tucumán (Granara de Willink \& Claps, 2003) y de P. citri en San Juan (Granara de Willink, 2004).

Planococcus ficus presenta comportamiento críptico y se localiza en troncos, brazos, brotes, hojas y racimos (Lentini et al., 2006). Inverna debajo del ritidoma de los troncos, fundamentalmente como hembras fecundadas o huevos en ovisacos (Varela et al., 2006). Luego de la apertura de ye- 
mas se pueden observar ninfas en la base de los brotes nuevos, y hacia fines de primavera y en verano se desplazan primero a las hojas y posteriormente a los racimos, donde las ninfas y adultos se ubican preferentemente en la zona calicinar y pedicelar (Merino \& Gerding, 2006). En otoño, cuando disminuye la calidad del follaje, migran hacia el tronco y se instalan debajo de su corteza (Walton \& Pringle, 2004; Lentini et al., 2006). Sus infestaciones se caracterizan por la variabilidad tanto dentro de los viñedos como entre ellos, así como de una temporada a otra (Geiger et al., 2001; Lentini et al., 2006; Quiroz Escobar, 2010; Salazar Proboste et al., 2010) donde pueden aumentar (Skinkis et al., 2009; Mohamed et al., 2012) o disminuir (Lentini et al., 2006) sus poblaciones. Según Le Clerg (1971) se puede establecer una relación entre la población de una plaga y las pérdidas que causa en el cultivo. Sin embargo, Geiger et al. (2001) mencionan que es riesgoso estimar el nivel de daño de una plaga con demasiada anticipación, ya que sus poblaciones pueden variar por la presencia de enemigos naturales o factores climáticos. Corroborando esto, los autores citados encontraron que la cantidad de racimos afectados por $P$. maritimus en cosecha está más relacionada con la cantidad de cochinillas presentes en pitones en inicio de verano que en inicio de primavera.

La presencia de grandes poblaciones afecta las características organolépticas de las uvas viníferas y la calidad de los mostos (Hidalgo Fernández \& Hidalgo Togores, 2011). Según Rius Garcia (2005) la disminución de la calidad de los mostos puede traducirse en un mayor riesgo de alteraciones en los vinos. Bordeu et al. (2012) no encontraron diferencias en la composición química básica de mostos de las variedades Chardonnay y Carmenere; sin embargo, en ambos cultivares los polifenoles totales disminuyeron con el incremento de racimos infectados, y la misma tendencia se observó en Carmenere con los antocianos y taninos.

Las cochinillas blandas (Hemiptera: Coccidae) están cubiertas por una capa de cera normalmente blanda, producen melaza y afectan distintas partes de las plantas, incluidas las raíces (Granara de Willink, 2004; Buchanan, 2008). Son de amplia distribución mundial y en Brasil, a Parthenolecanium persicae (Fabricius) y $P$ Parthenolecanium corni (Bouché) se las considera plagas de la vid (Botton et al., 2002; Afonso et al., 2004;). En la Argentina, en este mismo cultivo, Granara de Willink (2004) cita a $P$. persicae en Buenos Aires y Platinglisia noacki Cockerell en Corrientes.

Los objetivos del trabajo fueron determinar y caracterizar las cochinillas presentes en los viñedos y evaluar la incidencia de las cochinillas harinosas y su efecto sobre la calidad de los mostos.

\section{MATERIALES Y MÉTODOS}

\section{Determinación y caracterización de especies}

Se trabajó en Colonia Caroya $\left(31^{\circ} 2^{\prime} \mathrm{S}, 64^{\circ} 5^{\prime}\right.$ O), en viñedos de las variedades Chardonnay, Merlot y Malbec cultivadas bajo malla antigranizo, y Pinot noir e Isabella sin dicha malla.

Para observar la presencia, describir las especies y analizar aspectos de su comportamiento, entre 2006 y 2013 se recolectaron muestras de hojas, brotes del año, sarmientos y racimos afectados con cochinillas en distintos estados de desarrollo, desde yema hinchada hasta fin de caída de hojas (Pratt, 1996). En 2006 y 2007 se realizaron muestreos en estado fenológico de yema de invierno; para ello, a fines de abril, en 10 plantas de la variedad Chardonnay se eliminó el ritidoma en la parte basal, en dos sectores del tallo y en uno de las ramas, donde se colocaron trampas de cartón corrugado de $5 \mathrm{~cm}$ de alto (González et al., 2001). Las trampas se renovaron a mediados de julio, y la lectura final se realizó en septiembre. Entre mayo y agosto en 15 plantas de las variedades Chardonnay y Merlot se extrajeron trozos de 3-4 $\mathrm{cm}$ de ritidoma de cuatro zonas de cada planta, desde el injerto hasta $10 \mathrm{~cm}$ de profundidad en las raíces, en la mitad inferior y superior del tronco y en los brazos. Durante la primavera y verano, desde brotación hasta caída de hojas, se realizaron muestreos quincenales para detectar la presencia de cochinillas en brotes, hojas y racimos de las variedades Chardonnay, Merlot, Malbec e Isabella. En todos los casos los individuos presentes fueron separados de los órganos, colocados en alcohol 70\% y enviados al Instituto Miguel Lillo (Tucumán, Argentina) para su identificación.

\section{Incidencia de cochinilla harinosa}

En 2007 se evaluó la incidencia de P. ficus en racimos de la variedad Chardonnay. Se seleccionaron al azar 10 hileras y en cada una de ellas se evaluaron 10 racimos, uno cada cinco plantas; se determinó la incidencia como porcentaje de racimos afectados y la intensidad como porcentaje del raquis afectado (Walker, 1991). Los racimos se evaluaron de acuerdo a cinco grados de intensidad: $\sin$ daño, hasta $25 \%, 50 \%, 75 \%$ y $100 \%$ del raquis afectado.

En febrero de 2010 y 2011 se seleccionaron 15 viñedos distribuidos en toda la zona producto- 
ra implantados con distintas variedades. En cada viñedo se tomaron 10 sitios de muestreo de tres plantas cada uno. La presencia de cochinillas se evaluó en 5 brotes y 5 racimos de cada planta en estado fenológico de madurez. Se consideró planta y racimo afectado si al menos una de sus partes tenía cochinillas. Se evaluó la incidencia como porcentaje de plantas y de racimos afectados con $P$. ficus (Walker, 1991); con los datos de incidencia se calculó la frecuencia relativa como

$$
(f / N)^{*} 100
$$

donde: $f_{i}$ es la frecuencia absoluta (número de sitios con un determinado valor de incidencia) y $\mathrm{N}$ es el número total de sitios.

La relación entre incidencia en plantas y racimos se estudió mediante el uso de modelos lineales y no lineales (Campbell \& Madden, 1990; Walker, 1991). La bondad de los ajustes se analizó a través del coeficiente de determinación, el cuadrado medio del error (CME) y la significancia de los parámetros estimados (Di Rienzo et al., 2015).

\section{Efecto sobtre la calidad de los mostos}

Se tomaron muestras de racimos en las variedades Chardonnay y Pinot noir. Se clasificaron en sanos y dañados, y en Pinot noir estos últimos se separaron en cuatro grados de intensidad (Walker, 1991): hasta $25 \%, 25-50 \%, 50-75 \%$ y más de $75 \%$ de raquis afectado con cochinillas. Las bayas de cada clase de racimos se desprendieron del raquis y se dividieron por tamaño mediante un sistema de tamices. Sobre las bayas del tamaño más frecuente se midieron grados Brix, tenor zucarino, acidez total expresada en ácido tartárico, acidez volátil expresada en ácido acético y el pH; en las variedades tintas se determinó también antocianos (mg/baya) en hollejos. Los resultados se analizaron con ANAVA y las medias se compararon con la prueba de Duncan $(\alpha=0,05)$. Se utilizaron las técnicas normalizadas por el INV (2015) y el INTA (2004).

\section{RESULTADOS Y DISCUSIÓN}

\section{Determinación y caracterización de especies}

Se identificaron dos especies de cochinillas harinosas, Planococcus ficus (Signoret) y Pseudococcus viburni (Signoret) (Hemiptera: Pseudococcidae), y una especie de cochinilla blanda, Parthenolecanium persicae (Fabricius) (Hemiptera: Coccidae).

\section{Planococcus ficus (Signoret)}

Se observaron hembras y ninfas; ambas poseen cuerpo ovalado, convexo, rosado y segmentado. En coincidencia con lo descripto por Walton \& Pringle (2004) y Bentley et al. (2008), la hembra adulta mide aproximadamente $4 \mathrm{~mm}$ de longitud y $2 \mathrm{~mm}$ de ancho, está cubierta por secreciones pulverulentas blancas y una vez fecundada su abdomen se transforma en un ovisaco. El cuerpo de las ninfas es de forma semejante al de las hembras, pero en el primer estadio es de color amarillento y no está recubierto por secreciones pulverulentas.

En el 25\% de las trampas de cartón corrugado evaluadas en julio, se detectaron escasas hembras adultas en la parte basal, en el tronco medio y en las ramas; mientras que en septiembre no se detectaron cochinillas, ni restos en ninguno de los cuatro sectores. La escasa cantidad de individuos encontrados en estas trampas fue también observado en muestreos invernales en viñedos chilenos (Koplow \& Zaviezo, 2004), aunque allí es considerado un método útil para establecer movimientos migratorios de $P$. viburni en ciruelos (González et al., 2001).

En el ritidoma extraído durante el invierno se observó la presencia de hembras, ninfas y ovisacos con masas de huevos, adheridos a éste y al leño, lo que confirma que en Colonia Caroya las cochinillas invernan protegidas en los brazos de la vid, fundamentalmente en su cara inferior y en el tronco. Esto coincide con lo registrado para $P$. ficus en Mendoza (Becerra et al., 2006a), en California (Varela et al., 2006) y en Italia (Lentini et al., 2006); y para P. maritimus en California (Geiger et al., 2001). En estos lugares de invernación se observaron nacimientos de ninfas a partir de agosto en 2009 y 2014 y a partir de octubre en 2011.

En las hojas de las variedades Chardonnay, Merlot, Malbec e Isabella se observaron ninfas y adultos desde inicio de noviembre hasta caída de hojas en 2006 y 2007, lo que concuerda con lo registrado en Mendoza (Becerra et al., 2006a) y en California (Bentley et al., 2008); sin embargo, se ven desde más temprano que lo observado por Soler et al. (2002) en Uruguay, donde las cochinillas pasaron a las hojas a partir de enero (Soler et al., 2002).

En racimos de las variedades Chardonnay y Pinot noir se detectaron hembras con ovisaco y ninfas desde fines de noviembre, en cierre racimo, hasta febrero-marzo en madurez de cosecha. Se observó que tanto ninfas como adultos se ubican principalmente en el raquis, en los pedicelos y en la zona de unión de éstos con las bayas y en me- 
nor proporción en las bayas, lo que corrobora lo descripto por Merino \& Gerding (2006). Este comportamiento de la plaga hace que sea difícil de detectar cuando se encuentra en bajas densidades poblacionales y una vez cerrado el racimo, como fuera registrado también en otros viñedos de la Argentina y Perú (Becerra et al., 2006b).

\section{Pseudococcus viburni (Signoret)}

Se registró la presencia de hembras adultas de cuerpo ovalado, ligeramente convexo, color rosado recubierto de una fina secreción cerosa pulverulenta blanca, con filamentos marginales delgados y cortos, y filamentos caudales de una longitud que varía entre 1/4 y 1/2 del largo del cuerpo. Carece de banda dorsal oscura y produce ovisacos (Bentancourt \& Scatoni, 1999; González \& Volosky, 2006). La presencia de adultos con ovisaco y sin éste se observó en racimos próximos a los brazos y luego de la cosecha bajo el ritidoma de sarmientos de 1 o 2 años. Esta distribución de la plaga en la planta es semejante a lo descripto en Chile (González \& Volosky, 2006; Valenzuela, 2008). En Colonia Caroya sólo se la encontró en un viñedo de la variedad Merlot; esta baja incidencia coincide con lo observado en el sur de Brasil donde representa $8,6 \%$ de las especies presentes en vid (Morandi Filho, 2008) y se diferencia de lo que ocurre en Chile donde es una de las plagas más importantes en uva de mesa y vinífera (Koplow \& Zaviezo, 2004; González \& Volosky, 2006).

\section{Parthenolecanium persicae (Fabricius)}

Se observó la presencia de hembras que poseen forma globosa, de aproximadamente $7 \mathrm{~mm}$ de longitud por 3,5 a 4,5 mm de ancho; de color pardo a castaño y con estrías más oscuras en el dorso, lo que coincide con lo descripto por Botton et al. (2002) para esta especie. En invierno se observaron caparazones de hembras adheridos a sarmientos y troncos, que protegen masas de huevos amarillos durante el período de incubación. En primavera se registró la presencia de ninfas amarillas localizadas preferentemente en los sarmientos y a lo largo de las nervaduras en el envés de las hojas. Al igual que en Brasil (Afonso et al., 2004), esta especie fue observada en Colonia Caroya en plantas de $V$. vinifera y $V$. labrusca.

Todas las partes de las plantas afectadas por las especies descriptas, y como lo cita Granara de Willink (2004), estuvieron asociadas con la presencia de melaza y fumagina. También se observó intenso desplazamiento de distintas especies de hormigas; este movimiento se registró principalmente durante primavera y verano y coincide con lo descripto por González (1983) para P. persicae y Soler et al. (2002), Bentley et al. (2008), Becerra et al. (2006b) y Varela et al. (2006) para P. ficus y P. viburni. En numerosas ocasiones se pudieron detectar focos de cochinillas mediante la observación de las hormigas como indicadoras de su presencia, como lo evidenciaron también Soler et al. (2002).

\section{Incidencia de cochinilla harinosa}

En 2007 en la variedad Chardonnay la incidencia en racimos fue de $33 \%$ y la intensidad alcanzó $19,5 \%$ de raquis afectado. En 2010, P. ficus estuvo presente en $73 \%$ de los viñedos evaluados y en 2011 en $87 \%$ de los campos, lo que significa un aumento de $13 \%$ en un año.

En plantas la incidencia promedio aumentó de 17 a $28 \%$ de un año a otro; si bien este incremento no fue estadísticamente significativo $(P<0,1143)$ (Tabla 1), es importante agronómicamente debido a los daños directos sobre las plantas (Daane et al., 2008). Se observaron sitios con diferentes niveles de incidencia (Figura 1); la frecuencia de sitios con baja incidencia de cochinillas (0-25\%) fue de $69 \%$ en 2010 y disminuyó a $57 \%$ en 2011 , y la frecuencia de sitios con incidencia entre 25 y $75 \%$ se mantuvo relativamente estable (12-18\%) en am-

Tabla 1: Incidencia de $P$. ficus en plantas y racimos en viñedos de Colonia Caroya, Córdoba, en 2010 y 2011

\begin{tabular}{lcccc}
\hline Viñedo & \multicolumn{2}{c}{$\begin{array}{c}\text { Incidencia en } \\
\text { plantas }(\%)^{1}\end{array}$} & \multicolumn{2}{c}{$\begin{array}{c}\text { Incidencia en } \\
\text { racimos }(\%)^{2}\end{array}$} \\
\hline 1 & 2010 & 2011 & 2010 & 2011 \\
2 & 30,0 & 40 & 12 & 29,33 \\
3 & 23,3 & 16,66 & 13,3 & 5,33 \\
4 & 23,3 & 50 & 10 & 35,33 \\
5 & 0 & 0 & 0 & 0 \\
6 & 0 & 6.66 & 0 & 4,66 \\
7 & 16,7 & 13,33 & 5,3 & 7,33 \\
8 & 3,3 & 6,66 & 2 & 1,33 \\
9 & 3,3 & 3,33 & 0,6 & 0,66 \\
10 & 0 & 46,66 & 0 & 28,66 \\
11 & 30 & 56,66 & 17,3 & 44,66 \\
12 & 0 & 46,66 & 0 & 33,33 \\
13 & 40 & 46,66 & 14 & 26,66 \\
14 & 16,7 & 33,33 & 6,6 & 27,33 \\
15 & 30 & 0 & 13,3 & 0 \\
\hline Promedio & $17,1 \mathrm{a}$ & $28,44^{\mathrm{a}}$ & $7,6 \mathrm{a}$ & $18,53 \mathrm{~b}$ \\
\hline
\end{tabular}

1 Porcentaje de plantas afectadas en 30 plantas por viñedo. ${ }^{2}$ Porcentaje de racimos afectados en 150 racimos por viñedo. Prueba T para muestras Independientes. 
bos años. Mientras tanto, en este mismo período se observó un fuerte incremento (de 4 a 17\%) de sitios con 75 a $100 \%$ de plantas afectadas. En racimos se observó similar tendencia que en plantas (Figura 1); el porcentaje de sitios con nula o baja incidencia (0-20\%) de cochinillas disminuyó 16\% en 2011. Si bien la cantidad de sitios con incidencia entre $20-40 \%$ y $40-60 \%$ aumentó en 2011 , este incremento fue sólo de 2 y $3 \%$ respectivamente. En cambio la frecuencia de sitios con $60-80 \%$ de incidencia aumentó de 2 a $10 \%$ y se registraron por primera vez sitios con $80-100 \%$ de racimos afectados.

Estos resultados indican que las cochinillas en Colonia Caroya estuvieron presentes en la mayoría de los viñedos y que sus poblaciones incrementaron entre 2010 y 2011. Esto corrobora la variabilidad de las poblaciones observada en Chile (Salazar Proboste et al., 2010) y su rápido incremento en pocos años, según lo citado por Mohamed et al. (2012) y Daane et al. (2012). El aumento de un año a otro en la frecuencia de sitios con alta incidencia en plantas y racimos coincide con lo reportado por Skinkis et al. (2009) en Oregón, donde observaron altas infestaciones de cochinillas pocas temporadas después de su establecimiento en el cultivo. También concuerda con lo descripto por Mohamed et al. (2012) en Egipto, donde se convirtió en una plaga de importancia económica en pocos años en áreas recuperadas para la viticultura, como es el caso de la zona estudiada en este trabajo. Sin embargo, es importante considerar que este incremento puede ser parte de la variabilidad de las poblaciones de Pseudococidae observada por Lentini et al (2006) y Quiróz Escobar (2010), o de un comportamiento cíclico como el registrado por Geiger et al. (2001) a través de los años y entre viñedos. Estas variaciones pueden deberse a cambios en las prácticas culturales, a la presen- cia de enemigos naturales (Geiger et al., 2001) o a patrones climáticos (Geiger et al., 2001; Lentini et al., 2006), hecho que no se puede corroborar en el presente trabajo ya que se evaluaron las poblaciones de cochinillas durante dos años.

En el análisis de la relación entre la incidencia de cochinillas en plantas y en racimos se puede observar que las dos variables están positivamente asociadas $(p<0,0001)$ en $2010(r=0,96)$, en 2011 $(r=0,97)$ y en ambos años en conjunto $(r=0,95)$ (Figura 2); lo que indica que a medida que crece el porcentaje de plantas afectadas lo hace también el de racimos.

De acuerdo a la exploración gráfica de las curvas, y con valores de hasta $70 \%$ de plantas afectadas, se puede considerar que la relación entre incidencia en plantas y en racimos puede ser descripta por un modelo lineal o uno logístico (Figura 2). Esto se corrobora mediante el análisis del coeficiente de determinación $\mathrm{R}^{2}$, el CME (Tabla 2), la significancia de los parámetros estimados y la distribución de los residuos, para cada año en particular y en conjunto.

El ajuste obtenido por los dos modelos a los datos de 2011 indica una leve sobreestimación de la incidencia en racimos en valores bajos de incidencia en plantas y subestimación con valores altos. Esta tendencia también se observa cuando se analizan en conjunto los datos de 2010 y 2011 (Figura 2). El modelo logístico corrige estos ligeros desajustes y los valores estimados son más cercanos a los observados. El punto de máximo crecimiento en el modelo logístico indica este cambio de comportamiento en el porcentaje de incidencia en racimos, y dicho valor, que para el conjunto de años es de $36 \%$, corresponde al punto de inflexión de la curva.

Teniendo en cuenta los dos años evaluados por
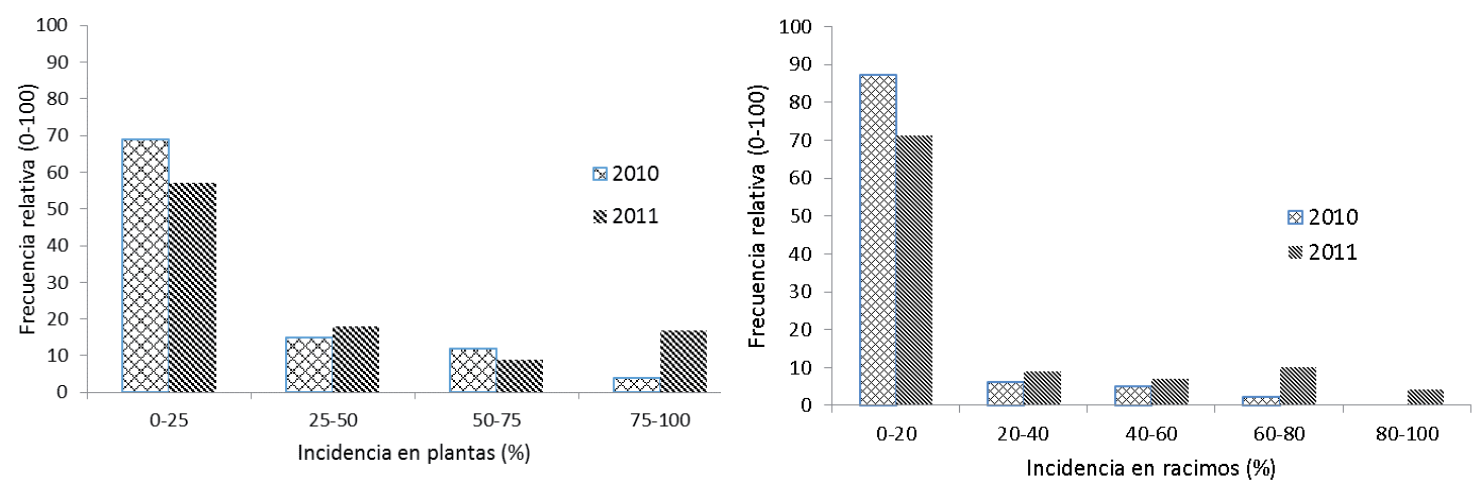

Figura 1: Frecuencia de la incidencia de P. ficus en plantas ${ }^{1}$ y racimos ${ }^{2}$ de vid en Colonia Caroya en 2010 y 2011.

1y2: Porcentaje de plantas y racimos afectados en 15 viñedos y 10 sitios de muestreo cada uno. 
A

B
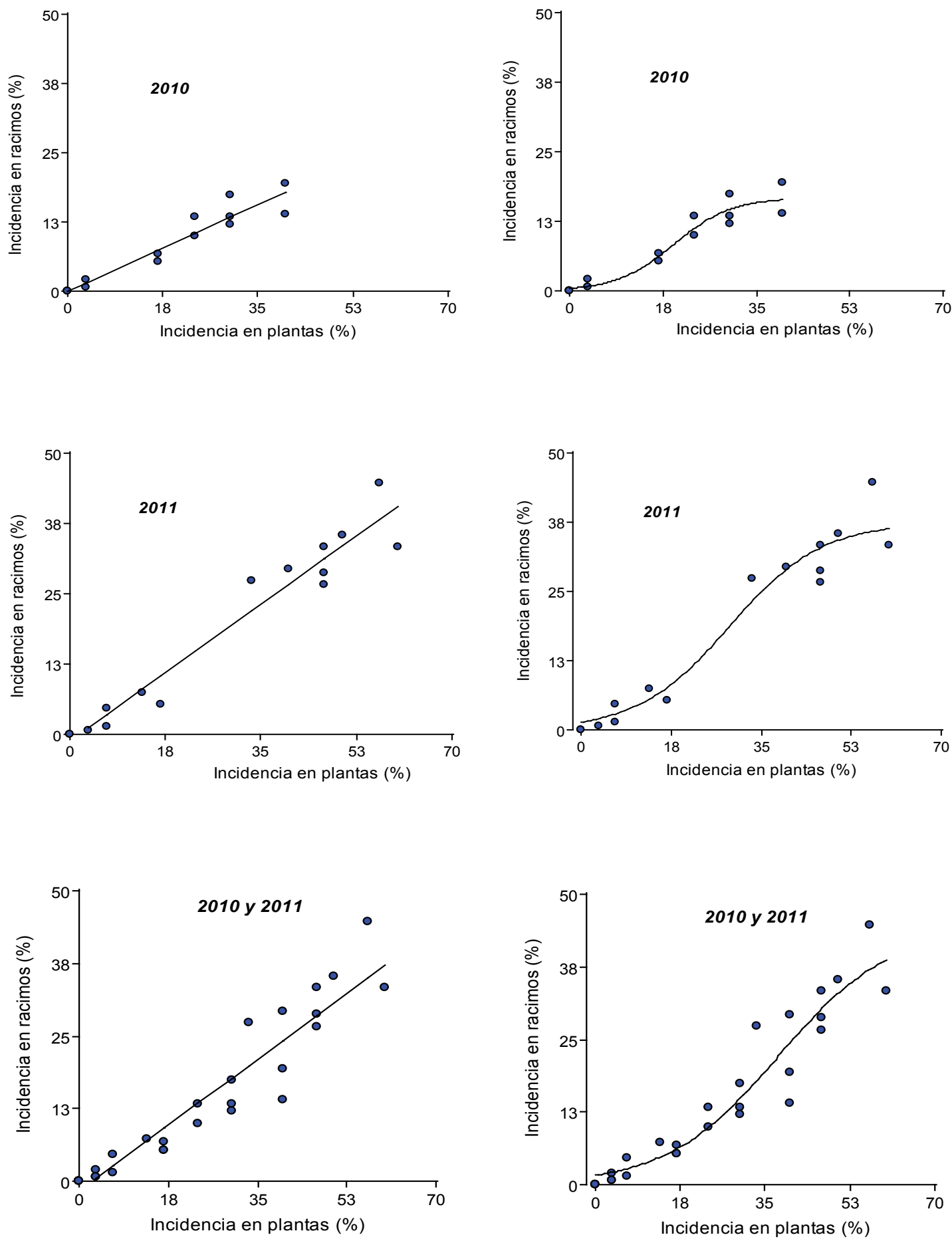

Figura 2: Incidencia de Planococcus ficus en plantas y en racimos de vid. Relación ajustada mediante modelos lineales (columna A) y logísticos (columna B). Cada punto corresponde al porcentaje promedio de plantas o racimos afectados en 10 sitios de muestreos en 15 viñedos 
Tabla 2: Modelos que mejor ajustan la relación entre incidencia de Planococcus ficus en plantas y en racimos de vid en dos años.

\begin{tabular}{|c|c|c|c|c|}
\hline & & \multirow[t]{2}{*}{ Modelo $^{2}$} & \multicolumn{2}{|c|}{ Bondad de ajuste $^{3}$} \\
\hline & & & $\mathrm{R}^{2}$ & CME \\
\hline \multicolumn{5}{|l|}{2010} \\
\hline & Lineal & $i r=-0,03+0,45 i p$ & 0,93 & 3,29 \\
\hline & Logístico & ir $=16,75 /\left(1+37,12^{*} \exp \left(-0,18^{\star} i p\right)\right)$ & 0,92 & 3,29 \\
\hline \multicolumn{5}{|c|}{$x_{2}$} \\
\hline & Lineal & $i r=-1,21+0,69 i p$ & 0,94 & 13,34 \\
\hline & Logístico & $i r=37,44 /\left(1+25,57^{*} \exp \left(-0,11^{*} i p\right)\right)$ & 0,94 & 15,17 \\
\hline \multicolumn{5}{|l|}{2010 y 2011} \\
\hline & Lineal & $i r=-1,66+0,65 i p$ & 0,90 & 16,34 \\
\hline & Logístico & $\mathrm{ir}=44,21 /\left(1+26,56^{*} \exp \left(-0,09^{*} \mathrm{ip}\right)\right)$ & 0,90 & 15,69 \\
\hline
\end{tabular}

${ }^{1}$ Datos de 10 sitios de muestreo en 15 viñedos de distintas variedades, ${ }^{2} \mathrm{ir}$ : incidencia en racimos (\%), ip: incidencia en plantas (\%), ${ }^{3}$ $\mathrm{R}^{2}$ coeficiente de determinación, CME cuadrado medio del error

separado y en conjunto, se puede considerar que los dos modelos ajustan a los datos de incidencia de cochinillas en racimos en función de la incidencia en plantas. Sin embargo la simplicidad del modelo lineal puede ser un factor determinante para su elección si se pretende estimar la cantidad de racimos afectados a partir de plantas con cochinillas.

Dentro de los escasos trabajos disponibles para estimar daños en racimos, Geiger et al. (2001) encontraron buena relación en California entre porcentaje de racimos infectados con Pseudococcus maritimus en cosecha y la cantidad de cochinillas presentes en pitones a mediados de estación (junio), y no así con las encontradas a principio de estación (marzo). Los datos obtenidos en junio les permitieron a dichos autores estimar que a partir de una cochinilla por pitón se podía esperar una pérdida de $25 \%$ de los racimos. Los resultados obtenidos en Córdoba, con datos de dos años, indican que, por ejemplo, a partir de $10 \%$ de plantas afectadas se podría esperar 4,9\% ० 3,7\% de racimos dañados a partir del modelo lineal o logístico, respectivamente. Si bien en el trabajo de Geiger et al. (2001) se pudo cuantificar el daño con dos meses de anticipación, la ventaja del presente trabajo radica en la practicidad de evaluar plantas, en lugar de pitones, con presencia de cochinillas durante la maduración de los frutos y con ello tener una aproximación de su daño en racimos. Según Le Clerg (1971) el análisis de regresión es una de las técnicas más útiles para traducir el daño de una plaga en pérdidas de rendimiento. En éste trabajo no se evaluó rendimiento pero se pudo establecer una relación entre daño en plantas y porcentaje de racimos dañados en cosecha.

\section{Efecto de $P$. ficus sobre la calidad de los mostos}

Se pudo observar que el tamaño representativo de las bayas fue el que quedó retenido en el tamiz de $11 \mathrm{~mm}$ en Chardonnay y Pinot noir y de 13 $\mathrm{mm}$ en Merlot, independientemente de los niveles de intensidad de raquis afectado con cochinillas (Tabla 3), lo que significa que la presencia de cochinillas no afectó el tamaño de las bayas en las distintas variedades.

En Chardonnay (tabla 4) los índices de calidad, $\mathrm{pH}$, grados Brix y acidez total se vieron poco afectados por la presencia de cochinillas en racimos, lo que coincide con lo observado en esta misma variedad por Bordeu et al. (2012), quienes no encontraron diferencias en la composición química de mostos provenientes de racimos afectados con distintos niveles de Pseudococcus spp. La acidez volátil aumentó de 0,06 a 0,18 en 2007 y de 0,06 a 0,07 en 2008 en mostos de racimos sanos y afectados, respectivamente. En este último año los mayores valores $(0,09)$ se observaron en racimos con más de $25 \%$ de raquis afectado. Con la presencia de $P$. ficus se manifestó un índice de glucano positivo en 2007 y 2008; y en este último año doble positivo con más de $25 \%$ de severidad, y altamente positivo con más de $75 \%$ de severidad. Si bien este compuesto es común de detectar en mostos y vinos de uvas con Botrytis cinerea, también se manifestó en el análisis de mostos producidos con uvas con cochinillas en niveles que pueden perturbar fuertemente su clarificación y filtración (Hidalgo Fernández \& Hidalgo Togores, 2011).

En las variedades tintas, Merlot y Pinot noir (Tabla 5), el pH aumentó de 3,45 en mostos provenientes de racimos sanos a 3,63 en los elaborados con racimos afectados, y según Rius (2005) 
Tabla 3. Porcentaje de bayas retenidas en tamices de distintos tamaño según niveles de intensidad de cochinilla harinosa en raquis. Variedades Chardonnay, Merlot y Pinot noir

\begin{tabular}{|c|c|c|c|c|c|c|c|c|c|c|c|c|c|c|c|}
\hline \multirow{3}{*}{ Intensidad ${ }^{1}$} & \multicolumn{5}{|c|}{ Chardonnay } & \multicolumn{6}{|c|}{ Merlot } & \multicolumn{3}{|c|}{ Pinot noir } & \\
\hline & \multicolumn{15}{|c|}{ Tamaño medio de tamiz (mm) } \\
\hline & 7 & 9 & 11 & 13 & 15 & 7 & 9 & 11 & 13 & 15 & 7 & 9 & 11 & 13 & 15 \\
\hline 0 & 0 & 5 & 75,5 & 19 & 0 & 0 & 0 & 36,5 & 54,5 & 9 & 5,3 & 7 & 47,5 & 38,7 & 1,4 \\
\hline $1-10$ & 0 & 2,5 & 81 & 15,7 & 0,5 & 0 & 0,5 & 32 & 58,5 & 9 & 20 & 5,5 & 38 & 35 & 1,5 \\
\hline $11-25$ & 0 & 6 & 76,3 & 17,2 & 0,5 & 0 & 1,5 & 41,5 & 47,5 & 10,5 & 21,9 & 4 & 39,1 & 33,3 & 1,6 \\
\hline $26-50$ & 0 & 12 & 73,4 & 14,6 & 0 & 0,5 & 1 & 35 & 44 & 19,5 & 14,9 & 1,9 & 39,6 & 42,1 & 1,4 \\
\hline+51 & 0 & 9,9 & 55,8 & 33,8 & 0,5 & 0 & 0 & 19,5 & 43 & 37,5 & 8 & 3,8 & 46,9 & 39,4 & 1,9 \\
\hline
\end{tabular}

${ }^{1}$ Porcentaje de raquis afectado con cochinilla

Tabla 4. Evaluación de calidad en mostos de la variedad Chardonnay provenientes de uvas sanas y con cochinillas

\begin{tabular}{lrrrrrrrrrcr}
\hline \multicolumn{1}{l}{ Intensidad $^{1}$} & \multicolumn{2}{c}{$\mathrm{pH}$} & \multicolumn{2}{c}{${ }^{\circ}$ Brix } & \multicolumn{2}{c}{ Acidez total } & \multicolumn{2}{c}{ Acidez volátil } & \multicolumn{2}{c}{ Glucano } \\
\cline { 2 - 11 } & 2007 & 2008 & 2007 & 2008 & 2007 & 2008 & 2007 & 2008 & 2007 & 2008 \\
\hline Sanos & 3,5 & 3,43 & 21 & 21 & 5,55 & 7,05 & 0,06 & 0,06 & - & - \\
Afectados & 3,5 & 3,46 & 20,6 & 20,5 & 5,7 & 7,04 & 0,18 & 0,07 & + & ++ \\
& $1-10$ & & 3,52 & & 21 & & 5,92 & & 0,06 & + \\
$11-25$ & & 3,35 & & 20 & & 7,5 & & 0,06 & ++ \\
$26-50$ & & 3,46 & & 21 & & 6,97 & & 0,09 & +++ \\
+51 & & 3,41 & & 20 & & 7,8 & & 0,09 & +++ \\
\hline
\end{tabular}

${ }^{1}$ Porcentaje de raquis afectado con cochinilla

valores de $\mathrm{pH}$ elevado producen mayor riesgo de alteraciones microbianas, reducen intensidad del color en vinos tintos y su habilidad de envejecimiento, entre otros efectos. Por otro lado, en la variedad Merlot los grados Brix (Rius, 2005) disminuyeron progresivamente de 21 en las sanas a 19 en las más afectadas, en tanto que en Pinot noir se mantuvieron entre 19 y 19,8. En las dos variedades los antocianos disminuyeron significativamente en los mostos elaborados a partir de racimos con más del $50 \%$ del raquis afectado, lo que también fue observado en mostos de uvas con mohos (Pszczolkowski et al., 2001) y en vinos Carmenere provenientes de racimos completamente infectados con cochinillas (Bordeu et al, 2012). En Pinot noir la acidez total aumentó de 6 a 6,52 en coincidencia con lo observado por Becerra (2011); la volátil tuvo un incremento mayor, de 0,09 en racimos sanos y menos afectados a 0,18 en los más afectados.

Los aumentos en el pH, la acidez total y volátil y la disminución en los grados Brix y antocianos registrados principalmente en los mostos elaborados con racimos más afectados con $P$. ficus en Colonia Caroya, son indicadores de una posible disminución de la calidad potencial de los vinos tintos producidos con estos mostos.

\section{AGRADECIMIENTOS}

A la Dra. M. C. Granara de Willink por la identificación de las distintas especies de

Tabla 5. Evaluación de calidad en mostos de las variedades Merlot y Pinot noir provenientes de racimos sanos y con cochinillas.

\begin{tabular}{ccccccccc}
\hline \multirow{2}{*}{$\begin{array}{c}\text { Intensidad } \\
(\%)\end{array}$} & $\mathrm{pH}$ & Brix & $\begin{array}{c}\text { Antocianas } \\
\text { mg/baya }\end{array}$ & $\mathrm{pH}$ & Brix & $\begin{array}{c}\text { Antocianas } \\
\text { mg/baya }\end{array}$ & $\begin{array}{c}\text { Acidez } \\
\text { total }\end{array}$ & $\begin{array}{c}\text { Acidez } \\
\text { volátil }\end{array}$ \\
\cline { 2 - 9 }$y$ & 3,45 & 21 & $1,21 \mathrm{~A}$ & 3,49 & 19 & $1,16 \mathrm{~A}$ & 6 & 0,09 \\
$1-10$ & 3,44 & 21 & $1,35 \mathrm{AB}$ & 3,51 & 19,2 & $1,14 \mathrm{~A}$ & 6 & 0,09 \\
$11-25$ & 3,4 & 20 & $1,32 \mathrm{AB}$ & 3,54 & 19,6 & $1,14 \mathrm{~A}$ & 6 & 0,09 \\
$26-50$ & 3,56 & 20 & $1,25 \mathrm{AB}$ & 3,55 & 19,4 & $1,14 \mathrm{~A}$ & 6,22 & 0,12 \\
+51 & 3,63 & 19 & $1,42 \mathrm{~B}$ & 3,63 & 19,8 & $0,85 \mathrm{~B}$ & 6,52 & 0,18 \\
\hline
\end{tabular}

${ }^{1}$ Porcentaje de raquis afectado con cochinilla

Medias con una letra común no son significativamente diferentes $(p>0,05)$ 
cochinillas. A la Secretaría de Ciencia y Técnica de la Universidad Nacional de Córdoba

\section{BIBLIOGRAFIA}

Afonso, A.P.; I. Teixeira, M. Botton, J.L. Faria y A. E. Loeck, 2004. Controle da cochonilha-parda Parthenolecanium persicae (Fabricius, 1776) (Hemiptera: Coccidae) na cultura da videira. Ciência Rural 34 (4): 985-989.

Becerra, V., 2011. Influencia del ataque de la "cochinilla harinosa de la vid" (Planococcus ficus), sobre las características enológicas y organolépticas de vinos cv. Malbec. INTA EEA Mendoza Centro de estudios de Enología. Informe №18/290305. 4 pp.

Becerra, V.; M. Gonzalez, M.E. Herrera y J.L. Miano, 2006a. Dinámica poblacional de Planococcus ficus Signoret (Hemiptera: Pseudococcidae) en viñedos. Mendoza (Argentina). Rev. FCA UNCuyo XXXVIII (1): 1-6.

Becerra, V.; M. Gonzalez, M.E. Herrera, J.L. Miano y J.L. Lanati, 2006b. Asociación entre cochinillas harinosas, enemigos naturales, malezas y hormigas en el cultivo de la vid. XXI Jornadas Fitosanitarias Argentinas Catamarca, pp. 109-110.

Bentancourt, C.M. y I.B. Scatoni, 1999. Pseudococcus viburni Chanchito blanco de los frutales o cochinilla harinosa. En guía de Insectos y Ácaros de importancia agrícola y forestal en el Uruguay. Universidad de la Republica, Fac. de Agronomía, Montevideo.

Bentley W. J.; L. G. Varela, F. G. Zalom, R. J. Smith, A. H. Purcell, P. A. Phillips, D. R. Haviland, K. M. Daane and M. C. Battany, 2008. Grape Mealybugs. Vine Mealybug. Updated 10/08. IC IPM OnLine

Bentley, W.J.; L Martin, R. Hanna, P. Schrader and D. Luvisi, 2000. Research factors influcing grape mealybug infestation in San Joaquin Valley table grapes. Viticulture Resarch Report 1999-2000, Vol 28. 11 pp.

Bordeu, E.; D.O. Troncoso, y T. Zaviezo, 2012. Influence of mealybug (Pseudococcus spp.) infested bunches on wine quality in Carmenere and Chardonnay grapes. International Journal of Food Science \& Technology, 47, 232-239.

Botton, M.; I. Teixeira y A.P. Alfonso, 2002. Bioecologia e Controle da cochonilha-parda Parthenolecanium persicae (Fabricius, 1776) (Hemiptera: Coccidae) na cultura da videira. Comunicado Técnico 44, Embrapa. $4 \mathrm{pp}$.

Buchanan, G. 2008. Biological control of grapevine scales. Final report to grape and wine research \& development corporation Project Number: DNR 03/01. Australia. 32 pp.

Bustos, J. 2004. Estiman los daños de una nueva plaga de las vides sanjuaninas. INTA Newsletter - № 302.
http://www.elsitioagricola.com/gacetillas/intaCom/ newsletter302.asp Consultada 31/8/2010

Campbell, C.L. y L.V. Madden, 1990. Temporal Analisis of Epidemics I: Description and Comparision of Disease Progress Curves. pg: 161-203. In: Introduction to Plant Disease epidemiology. John Wiley \& Sons, Inc. $532 \mathrm{pp}$.

Cordo, H.A.; G. Logarzo, K. Braun y O. Di Iorio, 2004. Catálogo de insectos fitófagos de la Argentina y sus plantas asociadas. USDA-ARG. Sociedad Entomológica Argentina, pp.284-394.

Daane K.M. and WJ Bentley. 2003. Mealybugs in California Vineyards. ID \& General Biology. University of California Cooperative Extension. http://www.vinemealybug.uckac.edu/ID-biology-damage.htm. Last edited: 03/21/2007 Consultada el 12/08/2010

Daane, K.; E. A. Weber and W. Bentley. 2004. Vine Mealybug: Formidable pest spreading through California Vineyards. Practical Winery \& Vineyard Magazine. http://www.practicalwinery.com/mayjune04/ mayjun04p60.htm (Consultada: 20/8/2010).

Daane, K.; M. Cooper, S. Triapitsyn, V.M. Walton, G. Yokota, D.R. Haviland, W. Bentley, K. Godfrey and L. Wunderlich, 2008. Vineyard managers and researchers seek sustainable solutions for mealybugs, a changing pest complex. California Agriculture 62(4):167-176.

Daane, K.; R.P.P. Almeida, V. A. Bell, J.T.S. Walker, M. Botton, M. Fallahzadeh, M. Mani, J. L. Miano, R. Sforza, V. M. Walton y T. Zaviezo, 2012. Biology and management of mealybugs in vineyards, pp. 271-307. In N. J. Bostanian, C. Vincent, R. Isaacs, (eds.), Arthropod Management in Vineyards: Pests, Approaches, and Future Directions. Springer, Dordrecht, The Netherlands.De Borbón, C.M; O. Gracia and G. Gómez Talquenca, 2004. Mealybugs and Grapevine LeafrollAssociated Virus 3 in Vineyards of Mendoza, Argentina. Am. J. Enol. Vitic. 55(3): 283-285.

Di Rienzo J.A.; F. Casanoves, M.G. Balzarini, L. Gonzalez, M. Tablada y C.W. Robledo. InfoStat versión 2015. Grupo InfoStat, FCA, Universidad Nacional de Córdoba, Argentina. <http://www.infostat.com.ar> Consultada 10/4/2016.

Fu Castillo, A.; J.L. Miranda Blanco, G. Osorio Acosta y J.L. Martínez Carrillo, 2004. Control químico de piojo harinoso Planococcus ficus Signoret (Homoptera: Pseudococcidae) en vid de mesa. Agricultura Técnica en México 30 (1): 101-105.

Geiger, C. A.; K. M. Daane, W. J. Bentley, G. Yokota and L. A. Martin, 2001. Sampling program for grape mealybugs improves pest management. California Agriculture 55(3): 19-27.

González R. y C. Volosky, 2006. Desarrollo estacional y estrategias de manejo de chanchitos blancos, Pseudococcus spp., en pomáceas, uvas de mesa y vid 
vinífera (Hemiptera: Pseudococcidae). Revista Frutícola 27:37-47

González, R. H.; J. Poblete y G. Barría, 2001. El chanchito blanco de los frutales en Chile, Pseudococcus viburni (Signoret), (Homoptera: Pseudococcidae). Rev. Frutícola 22:11-21.

Gonzalez, R.H., 1983. Conchuela grande café de la vid Parthenolecanium persicae (Fabricius) (Homoptera: Coccidae). En Manejo de plagas de la vid. Chile: Facultad de Ciencias Agrarias, Veterinarias y Forestales. Pp. 51-55.

Granara de Willink, M. C., 2004. Homoptera: Coccidae y Pseudococcidae. En Cordo, H.A.; G. Logarzo, K. Braun y O. Di lorio. 2004. Catálogo de insectos fitófagos de la Argentina y sus plantas asociadas. USDAARG. Sociedad Entomológica Argentina. 720 pp.

Granara de Willink, M.C. y L. Claps, 2003. Cochinilas (Hemiptera: Coccoidea) presentes en plantas ornamentales de Argentina. Neotropical Entomology 32 (4): 625-637.

Granara de Willink, M.C.; I.B. Scatoni, A.L. Terra y M.I. Frioni, 1997. Cochinillas harinosas (Homoptera- Coccoidea- Pseudococcidae) que afectan plantas cultivadas y silvestres en Uruguay. Agrociencia 1: 96-99.

Hidalgo Fernández-Cano, L. y J. Hidalgo Togores, 2011. Tratado de viticultura. Vol 1 y 2, 4ª Edición. Ed. Mundi Prensa. Madrid, España. 2096 pp.

INTA (Instituto de Nacional de Tecnología Agropecuaria). 2004. Protocolo de técnicas analíticas. Ed. INTA Mendoza. 120 pp.

INV (Instituto Nacional de Vitivinicultura). 2015. http:// www.inv.gov.ar/inv_contenidos/pdf/estadisticas/anuarios/2015/Registro15.pdf. Consultada 18/4/2016.

INV (Instituto Nacional de Vitivinicultura). 1970. Manual de técnicas analíticas del I.N.V. Método oficial argentino. 150pp.

Koplow, C. y T. Zaviezo, 2004. Evaluación de distintas estrategias de monitoreo de chanchito blanco, Pseudococcus viburni (Signoret), (Hemiptera: Pseudococcidae) en vid Fac. Agronomía e Ing. Forestal. Pontificia Universidad Católica de Chile. Pp. 38-50.

Le Clerg, E.L., 1971. Field experiments for assessment of crop losses. Crop Loss assessment methods. FAO. Manual on the evaluation and prevention of losses by pests, diseases and weeds. Ed. L. Chiarappa. Pag.2.1/1-2.1./10.

Lentini, A; G. Serra, S. Ortu, G. Delrio, 2006. Seasonal abundance and distribution of Planococcus ficus on grape vine in Sardinia. IOBC/wprs Bulletin 36: 267272.

Merino L.M. y M. Gerding, 2006. Avances en el Control Biológico del chanchito blanco de la vid con hongos entomopatógenos
http://www.inia.cl/medios/quilamapu/pdf/bioleche/BOLETIN135.pdf. Consultada 30/4/2010

Miano, J. L.; M. Lannoy y M. Rodriguez Rolfi, 2014. Manejo integrado de plagas en viñedos, desafío sólo para amantes de la sustentabilidad. Obtenido de 37th World Congress of Vine and Wine and 12th General Assembly of the OIV:: http://dx.doi.org/10.1051/oivconf/201405005. Consultada 30/4/2015

Mohamed, G. H.; S.A. Badr y S.M. El-Amir, 2012. The efficiency of different materials for controlling vine mealybug, Planococcus ficus (Hemiptera: Pseudococcidae) on grapes during the period of investigation. Egypt. Acad. J. Biolog. Sci. 4(1), 69- 75.

Morandi Filho, W. J., 2008. Cochonilhas-farinhentas associadas à videira na Serra Gaúcha, bioecologia e controle de Planococcus citri (Risso, 1813) (Hemiptera: Pseudococcidae). Tese apresentada ao Programa de Pósgraduação em Fitossanidade da Universidade Federal de Pelotas, Brasil. 90 pp.

Pearson, R.C. y A.C. Goheen, 1996. Plagas y enfermedades de la vid. The American Phytopatologycal Society. Ediciones Mundi Prensa, Madrid. 91 pág.

Pratt, C., 1996. Estructura de la vid y fases de crecimiento. En: Plagas y Enfermedades de la Vid. Eds. R.C. Pearson y A.C. Goheen, Ediciones Mundi-Prensa, Madrid, España, pp. 3-7.

Pszczolkowski, P.H.; B.A. Latorre y C. Ceppi Di Lecco, 2001. Efectos de los mohos presentes en uvas cosechadas tardíamente sobre la calidad de los mostos y vinos Cabernet Sauvignon. Cien. Inv. Agr. 28(3): 157-163.

Quiróz Escobar, C., 2010. Introducción. En Salazar A.; M. Gerding, P. Luppichini, R. Ripa, R., P. Larraín, T. Zaviezo y P. Larral. Biología, manejo y control del chanchitos blancos. Boletín INIA N²04.

Rius Garciía, X., 2005. Apuntes de viticultura Australiana. Ed. Agro Latino. Barcelona España. 445 pp.

Salazar A.; M. Gerding, P. Luppichini, R. Ripa, R., P. Larraín, T. Zaviezo y P. Larral, 2010. Biología, manejo y control de canchitos blancos. Boletín INIA N² 204. 60 pp

Skinkis P.A.; A.J. Dreves, V.M. Walton y R.R. Martin, 2009. Field Monitoring for Grapevine Leafroll Virus and Mealybug in Pacific Northwest Vineyards. Oregon State University Extension Service EM 8985. Oregon, USA. 6 pp.

Soler, R.; I. Scatoni y S. Núñez, 2002. Bioecología y estrategia de control del "Chanchito blanco" de la vid en la zona sur del Uruguay. Período de investigación: 1997-2001. Convenio INIA-FUCREA. 16pp.

Toledo Paños, J., 2004. Melazo o cochinilla algodonosa (Pseudococcus citri, Risso). En Los parásitos de la vid. Estrategias de protección razonada. Coedición 
MAPA -Mundi-Prensa. 5a Edición. España, pp 58-61. Trjapitzin, S.V. and V.A. Trjapitzin, 1997. Parasites of Mealybugs (Homoptera, Pseudococcidae) on Cultivated Grapes in Argentina, with Description of a New Species of the Genus Aenasius Walker (Hymenoptera, Encyrtidae). http://www.maik.ru/abstract/enteng/99/ enteng0386_abstract.pdf. Consultada 28/9/2010.

Valenzuela, F. M., 2008. Evaluación de un programa de control biológico de Pseudococcus viburni (Signoret) en Vitis vinífera (Linneo) en el valle de Casablanca. 59 pp. http://ucv.altavoz.net/prontus_unidacad/site/ artic/20080811/asocfile/20080811164309/fmercadal. pdf. Consultada 29/9/2009.

Varela L.G.; R.J. Smith, M. Battany and W. Bentley, 2006.
Which mealybug is it, why should you care? Practical Winery \& Vineyard Magazine. 6pp. http://www.practicalwinery.com/janfeb06/janfeb06p37.htm Consultada 29/9/2009.

Walker, P.T., 1991. Measurement of insect pest populations and injury. Pag. 19-29. En Crop loss assessment and pest management Editor Teng, P.S. APS press The American Phytopathological Society, Saint Paul Minnesota.

Walton, V. M. and K. L. Pringle, 2004. Vine mealybug, Planococcus ficus (Signoret) (Hemiptera: Pseudococcidae), a Key Pest in South African vineyards. A Review S. Afr. J. Enol. Vitic., 25 (2): 54-62. 